\title{
Expression of angiogenic factors in hepatocarcinogenesis: Identification by antibody arrays
}

\author{
TAKAKO NOMURA $^{1}$, ASAHIRO MORISHITA ${ }^{1}$, GONG JIAN $^{1}$, SHIMA MIMURA ${ }^{1}$, KIYOHITO KATO ${ }^{1}$, \\ KEI NOMURA ${ }^{1}$, JOJI TANI ${ }^{1}$, HISAAKI MIYOSHI ${ }^{1}$, HIROHITO YONEYAMA ${ }^{1}$, TEPPEI SAKAMOTO ${ }^{1}$, \\ KOJI FUJITA ${ }^{1}$, EMIKO MAEDA ${ }^{1}$, HIDEKI KOBARA ${ }^{1}$, HIROHITO MORI ${ }^{1}$, \\ HISAKAZU IWAMA $^{2}$ and TSUTOMU MASAKI ${ }^{1}$ \\ ${ }^{1}$ Department of Gastroenterology and Neurology, ${ }^{2}$ Information Technology Center, Kagawa University \\ School of Medicine, Miki-cho, Kita-gun, Kagawa 761-0793, Japan
}

Received May 1, 2013; Accepted June 21, 2013

DOI: 10.3892/or.2013.2674

\begin{abstract}
Angiogenesis plays a pivotal role in the progression and metastasis of hepatocellular carcinoma (HCC). However, the expression of a wide range of angiogenic factors remains obscure in HCC. The purpose of the present study was to determine the expression of various angiogenic factors related to hepatocarcinogenesis. We examined the expression of 19 angiogenic factors using antibody arrays in human tissues of various liver diseases, including HCC. We also studied the expression of 19 angiogenic factors in the human HCC cell lines PLC/PRF/5, Hep 3B, HuH7, HLE, HLF and Li-7 and the normal hepatocyte cell line ACBRI3716. In human tissues, although the expression of acidic fibroblast growth factor (aFGF) was found to increase from normal liver to chronic hepatitis, its expression remained unchanged in the transition from chronic hepatitis to HCC. Vascular endothelial growth factor (VEGF) was elevated in liver cirrhosis, but the amounts remained unchanged in the transition from liver cirrhosis to HCC. In contrast, either interleukin-8 (IL-8) or basic fibroblast growth factor (bFGF) was upregulated in HCC. In the HCC cell lines PLC/PRF/5, Hep 3B and HuH-7, the expression of IL-8 was elevated. Although IL-8 was not elevated, bFGF was upregulated in the other HCC cell lines HLE, HLF and Li-7. Thus, either IL-8 or bFGF was upregulated in HCC cell lines and in HCC tissue samples. These data suggest that the upregulation of either IL-8 or bFGF is closely related to the transition from liver cirrhosis into HCC. Therefore, the analysis of the expression of these cytokines using protein arrays may identify novel therapies for individual patients with HCC.
\end{abstract}

Correspondence to: Professor Tsutomu Masaki, Department of Gastroenterology and Neurology, Kagawa University School of Medicine, 1750-1 Ikenobe, Miki-cho, Kita-gun, Kagawa 761-0793, Japan

E-mail: tmasaki@med.kagawa-u.ac.jp

Key words: hepatocellular carcinoma, angiogenesis, basic fibroblast growth factor, interleukin-8

\section{Introduction}

Liver cancer is the third leading cause of cancer deaths worldwide and was responsible for 696,000 deaths in 2008 (1). HCC, which is the main type of primary liver cancer, is more difficult to treat than many other cancers because HCC is generally induced as a consequence of underlying liver diseases, such as chronic hepatitis and liver cirrhosis. Surgical resection and percutaneous local ablation are curative treatments, but these applications are limited to HCC patients with well-preserved liver function; metastasis is quite common (2). In addition, postoperative recurrence is a persisting issue (3). Liver transplantation is another curative treatment of HCC with liver cirrhosis, but the lack of donor organs is the main restricting factor for liver transplantations and contributes to prolonged waiting time (4). Systemic therapies have not been shown to be effective for advanced HCC. Thus, there is a strong demand for new curative approaches to HCC.

Angiogenesis plays an important role in the proliferation and metastasis of solid tumors (5). HCC is a hypervascular tumor characterized by vigorous neovascularization. Neovascularization is pivotal in the growth and progression of HCC and increases during the early phase of HCC development (6). Without neovascularization, tumors cannot become larger than a few cubic $\mathrm{mm}$ in size, and they remain in a state of tumor dormancy $(7,8)$. Thus, antiangiogenic treatments for solid tumors have been explored as a new strategy to suppress tumor growth and progression.

Several studies have shown that the expression of many angiogenic factors is closely related to the growth and metastasis of HCC $(9,10)$. However, the expression and interaction of a wide range of angiogenic factors remains obscure in HCC. In the present study, we examined the expression of various angiogenic factors related to hepatocarcinogenesis.

\section{Materials and methods}

Tissue samples. Human tissue samples of HCC, cholangiocellular carcinoma (CCC), colorectal cancer and esophageal cancer with the adjacent tissues were obtained during surgery or liver biopsy from 9 patients ( 7 males and 2 females; mean 
Table I. Profile of the TranSignal angiogenesis antibody array.

\begin{tabular}{lllllllll}
\hline & 1 & 2 & 3 & 4 & 5 & 6 & 7 & 8 \\
\hline A & pos & pos & pos & pos & pos & pos & pos & pos \\
B & Ang & Ang & IL-1 $\alpha$ & IL-1 $\alpha$ & aFGF & aFGF & IFN- $\gamma$ & IFN- $\gamma$ \\
C & G-CSF & G-CSF & IL-1 $\beta$ & IL-1 $\beta$ & bFGF & bFGF & IL-12 & IL-12 \\
D & HGF & HGF & IL-6 & IL-6 & TNF- $\alpha$ & TNF- $\alpha$ & IP-10 & IP-10 \\
E & Leptin & Leptin & IL-8 & IL-8 & TGF- $\alpha$ & TGF- $\alpha$ & TIMP-1 & TIMP-1 \\
F & VEGF & VEGF & PIGF & PIGF & neg & neg & TIMP-2 & TIMP-2 \\
\hline
\end{tabular}

pos, positive control; neg, negative control. All other abbreviations are standard.

age, $70.8 \pm 4.4$ years; range, $51-92$ years). None of the patients received any chemotherapy or radiotherapy before surgery. The use of human specimens was approved by the Human Subjects Committee of Kagawa University School of Medicine.

Cell culture. PLC/PRF/5, Hep 3B, HuH7, HLE, HLF and Li-7 cells, kind gifts from the Health Science Research Resource Bank (Osaka, Japan) and the Cell Resource Center for Biomedical Research (Institute of Development, Aging and Cancer, Tohoku University, Miyagi, Japan), were used as human HCC cell lines. ACBRI3716 cells were obtained from the Applied Cell Biology Research Institute (Kirkland, WA, USA) and used as normal human hepatocytes. These cells were cultured in Dulbecco's modified Eagle's medium (Gibco BRL, Tokyo, Japan) supplemented with $10 \%$ fetal calf serum (Gibco BRL), $100 \mu \mathrm{g} / \mathrm{ml}$ penicillin and $100 \mu \mathrm{g} / \mathrm{ml}$ streptomycin at $37^{\circ} \mathrm{C}$ under $5 \% \mathrm{CO}_{2}$ in air.

Preparation of protein from tissues and cell lines. We thawed and homogenized tissue samples with PRO-PREP ${ }^{\mathrm{TM}}$ (iNtRON Biotechnology, Inc., Seoul, Korea) solution containing $1 \mathrm{mM}$ of each of the protease inhibitors phenylmethylsulfonyl fluoride and ethylenediaminetetraacetic acid, $1 \mu \mathrm{M}$ each of pepstatin $\mathrm{A}$ and leupeptin and $0.1 \mu \mathrm{M}$ aprotinin. The homogenate was centrifuged at $13,000 \mathrm{x}$ g for $5 \mathrm{~min}$ after incubation for 20-30 min on ice. Equivalent amounts of the supernatant from tissue samples were used for antibody arrays. Liver-infiltrating mononuclear cells (LMNCs) were separated by Ficoll-Hypaque density gradient centrifugation (Histopaque; Sigma-Aldrich Co., St. Louis, MO, USA). We harvested the cell pellet by centrifuging at $13,000 \mathrm{x} \mathrm{g}$ for 10-20 sec. The pellet was mixed well with PRO-PREP solution and incubated on ice for 10-20 min. The cell lysate was centrifuged at 13,000 x g for $5 \mathrm{~min}$ after incubation. Equivalent amounts of the supernatant from cell lines were used for antibody arrays.

Protein assay. The protein concentration was determined according to the Bradford dye-binding assay (11).

Antibody array to screen angiogenic factors. To detect angiogenic factors, we used the TranSignal ${ }^{\mathrm{TM}}$ Angiogenesis Antibody Array (Panomics Inc., Redwood, CA, USA), in which 19 different antibodies against angiogenic factors are immobilized at predetermined positions on the membrane
(Table I). The angiogenesis antibody array was based on the sandwich ELISA method for detecting protein (12). The assay was performed following the manufacturer's protocol. The samples of tissue extracts and cell lysates were incubated with the array membranes at the same concentration of $4 \mathrm{mg} / \mathrm{ml}$. Then, biotin-labeled detection antibodies were added to the array membranes. The antibody-protein complexes on the array membranes were visualized using streptavidin-HRP to determine which active angiogenic factors were present in the samples. Immunoreactive proteins were visualized with an enhanced chemiluminescence detection system (Amersham Japan Co., Tokyo, Japan) on a radiograph film. The array data were normalized using positive control signal. The average local background signal was subtracted from average signal intensity of duplicated spots of each antibody.

\section{Results}

Expression of angiogenic factors in normal liver and various liver diseases. We used angiogenesis antibody arrays to investigate which angiogenic factors were expressed in normal human liver and various liver diseases. The expression of angiogenin was detected in normal liver (Fig. 1A). The expression of aFGF was found to increase from normal liver to chronic hepatitis (Fig. 1B). Furthermore, VEGF was upregulated from chronic hepatitis to liver cirrhosis (Fig. 1C). Noteworthy, two different expression patterns of angiogenic factors were detected in HCC. An IL-8 overexpression with weak bFGF expression pattern, or a bFGF overexpression pattern was observed. Therefore, IL-8 or bFGF was involved in the development of HCC from liver cirrhosis (Fig. 1D).

Expression of angiogenic factors in normal human hepatocyte and HCC cell lines. We also used angiogenesis antibody arrays to examine which angiogenic factors were expressed in the normal human hepatocyte and various HCC cell lines. The expression of angiogenin was not detected from the normal hepatocyte cell line or from various HCC cell lines (Fig. 2A). The expression of IL-8 was elevated in the HCC cell lines PLC/PRF/5, Hep 3B and HuH-7. In contrast, although the expression of IL-8 was not detected, bFGF was strongly expressed in the other HCC cell lines HLE, HLF, and Li-7, compared to the normal hepatocyte cell line (Figs. 2A and B). Thus, either IL- 8 or bFGF was upregulated in HCC cell lines. 

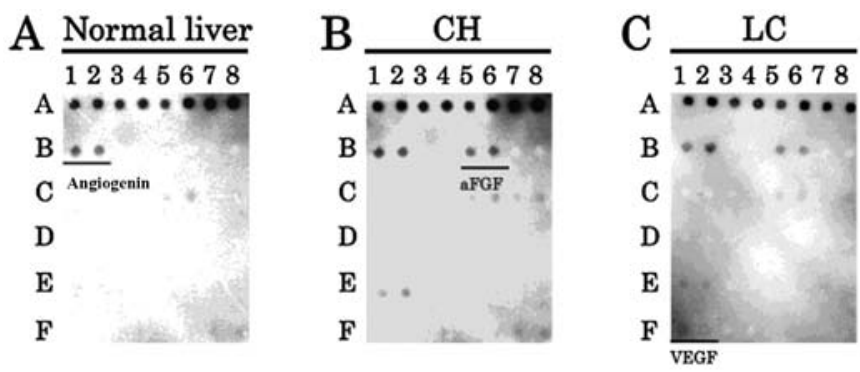

\section{$\mathrm{D}$}

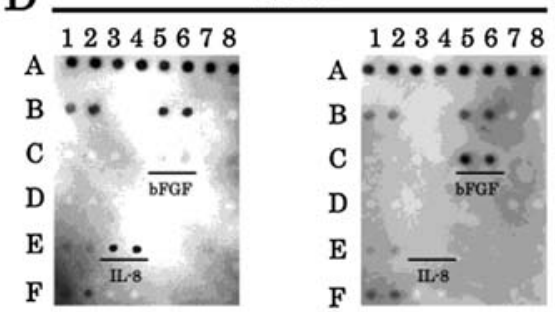

Figure 1. The expression patterns of angiogenic factors in normal human liver and various liver diseases. Protein from normal liver tissue (A), chronic hepatitis tissue (B), liver cirrhosis tissue (C) and HCC tissue sections from human livers (D) was extracted by homogenization. Angiogenesis antibody arrays were incubated with tissue extracts. The expression of aFGF was found to increase in the transition from normal liver to chronic hepatitis, and VEGF was upregulated between chronic hepatitis and liver cirrhosis. Furthermore, either IL- 8 or bFGF was upregulated in the development of $\mathrm{HCC}$ from liver cirrhosis.

Expression of angiogenic factors in HCC with and without liver-infiltrating mononuclear cells (LMNCs). To evaluate the involvement of LMNCs in the expression of angiogenic factors in HCC tissues, we examined the samples of HCC tissues with or without LMNCs by angiogenesis antibody arrays. The increased expression of IL-8 can be observed in HCC samples with or without LMNCs (Fig. 3). These results suggested that IL-8 was actively produced not only in LMNCs but also in HCC cells.

Expression of angiogenic factors in various malignant gastrointestinal tumors. In order to determine angiogenic factors in other malignant tumors, IL-8 was examined in cholangiocellular carcinoma (CCC), colorectal cancer and esophageal cancer samples. Notably, IL-8 was strongly expressed in the cancerous tissues, but not in non-cancerous tissues from each organ (Fig. 4). These results were similar to the data from the liver. Therefore, IL- 8 is suggested to also play an important role in gastrointestinal cancers (Fig. 5).

\section{Discussion}

Angiogenesis is a prime regulator of tumor growth, and anti-angiogenic factors are likely to become an important component of therapeutic strategies (13). It has been suggested that angiogenesis is an early event in tumorigenesis (14). HCC is a typical hypervascular tumor that is characterized by neovascularization. Many angiogenic factors have been studied in $\mathrm{HCC}$, and several anti-angiogenic therapies have been tested in animals and patients $(15,16)$. Of note, in our present study, the expression of either IL-8 or bFGF was enhanced in human HCC tissues and hepatocellular carcinoma cell lines (Figs. 1

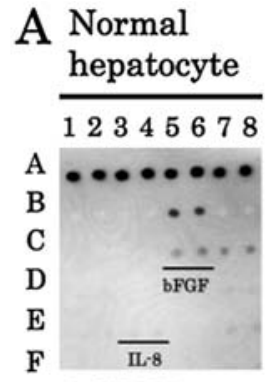

ACBRI3716

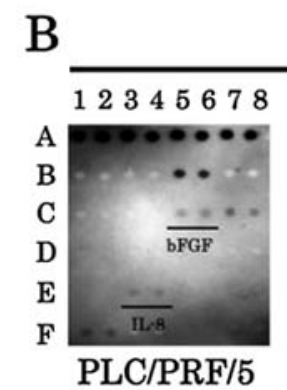

HCC cell lines

12345678
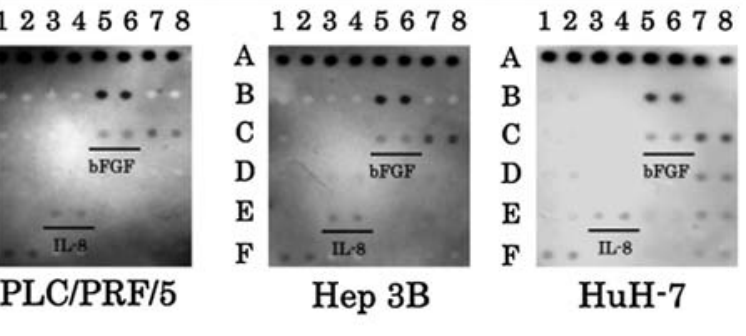

$\mathrm{HuH}-7$

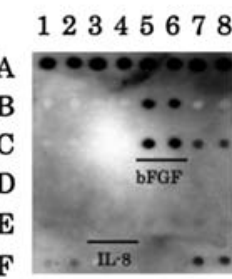

HLE

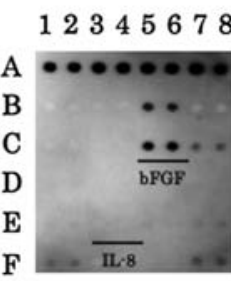

HLF

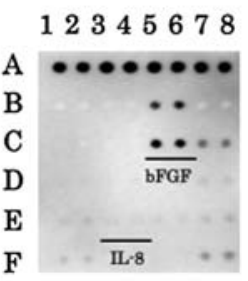

Li-7
Figure 2. The expression patterns of angiogenic factors in the normal human hepatocyte cell line and various HCC cell lines (A). Angiogenesis antibody arrays were incubated with cell lysates. The expression of IL-8 was elevated in HCC cell lines PLC/PRF/5, Hep 3B and HuH-7. Although the expression of IL-8 was not detected, bFGF was strongly expressed in HCC cell lines HLE, HLF, and Li-7, compared to the normal hepatocyte cell line (A and B). Thus, either the expression of IL-8 or bFGF was elevated in HCC cell lines. $\mathrm{CH}$, chronic hepatitis; LC, liver cirrhosis.

and 2). IL-8, a member of the CXC chemokine family, is a potent angiogenic stimulator $(17,18)$. IL-8 has recently been shown to contribute to human cancer progression through its potential functions as a mitogenic, angiogenic, and motogenic factor (19). Angiogenesis and tumor growth are inhibited by the downregulation or neutralization of IL-8 in several tumor models $(20,21)$. In addition, we demonstrated that IL- 8 was produced from human HCC tissues without LMNCs (Fig. 3). Moore et al have reported that some HCC cell lines secrete IL-8 (21). In addition, the serum level of IL-8 increased prior to the development of HCCs and increased further after the tumors appeared (22). Surprisingly, IL-8 was also enhanced in other cancer tissues, such as cholangiocarcinoma, colon cancer and esophageal cancer (Fig. 4). These results suggest that IL- 8 may be closely involved in carcinogenesis and the progression of various cancers, including HCC.

bFGF is a potentially important angiogenic stimulator. The serum level of bFGF is correlated with tumor invasiveness and postoperative recurrence in HCC (23). In the present study, our data also demonstrated that bFGF was upregulated in human HCC samples and some HCC cell lines (Figs. 1 and 2). Yoshiji et al have reported that bFGF synergistically augments 
$\mathrm{HCC}$

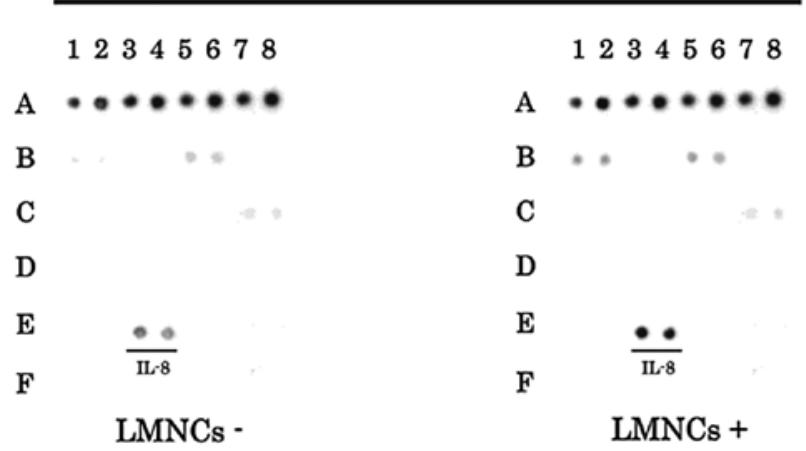

Figure 3. The expression patterns of angiogenic factors in the human tissues of HCC. Protein from HCC tissue sections from human liver tissues was extracted by homogenization, and liver-infiltrating mononuclear cells (LMNCs) were separated by Ficoll-Hypaque density gradient centrifugation. Angiogenesis antibody arrays were incubated with tissue extracts of HCC with or without LMNCs. The increased expression of IL-8 can be observed from HCC with or without LMNCs.

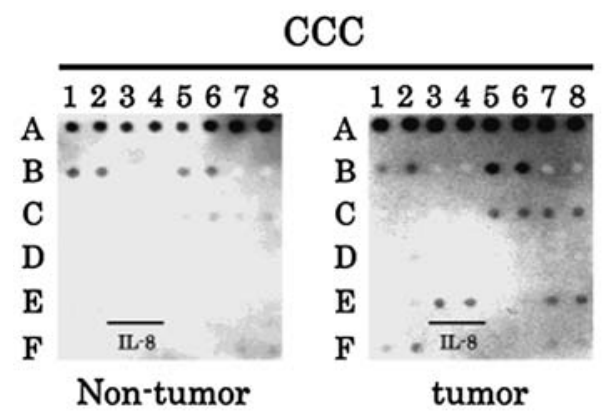

\section{Colorectal cancer}

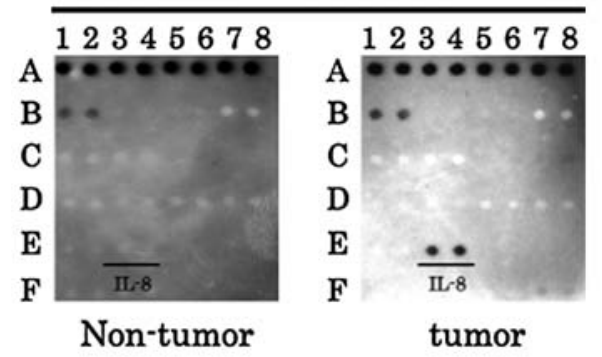

\section{Esophageal cancer}

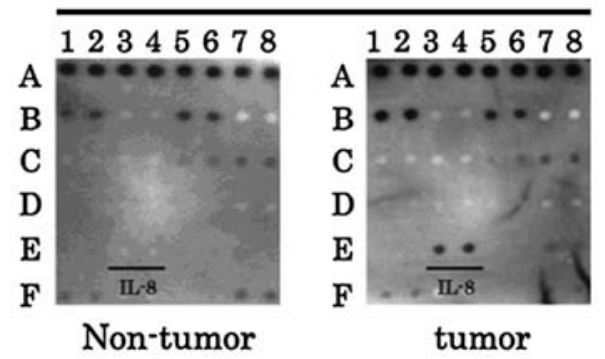

Figure 4. The expression patterns of angiogenic factors in non-cancerous and cancerous tissues from the gastrointestinal tract. Angiogenesis antibody arrays were incubated with each tissue extract. Protein from non-cancerous and cancerous tissue sections from human liver, colon and esophagus was extracted by homogenization. The expression of IL- 8 was elevated in the $\mathrm{CCC}$, colorectal cancer and esophageal cancer samples, compared to the non-cancerous tissues from each organ.

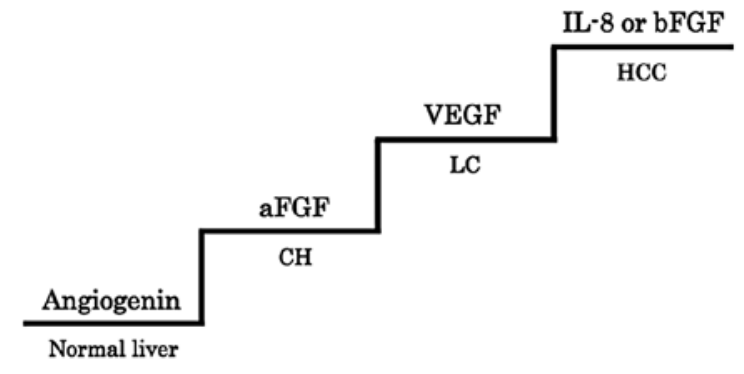

Figure 5. The expression of angiogenic factors in the development of HCC from normal liver. aFGF was increased in the transition from normal liver to chronic hepatitis, and VEGF was upregulated in the transition from chronic hepatitis to liver cirrhosis. The increase of either IL- 8 or bFGF occurs during the transition from liver cirrhosis into HCC. The suppression of IL- 8 and bFGF may offer a novel strategy to overcome the development and progression of HCC.

VEGF-mediated HCC development and angiogenesis, partly by the induction of VEGF through KDR/Flk-1 (24). These studies strongly support our results that bFGF involvement in angiogenesis is important for HCC development.

However, there is little documentation of the relationship between IL- 8 and bFGF to date. Our studies demonstrated two patterns for the expression of angiogenic factors in HCC cell lines and tissues: i) high IL-8 and low bFGF expression and ii) high bFGF and no IL-8 expression. Angiogenic growth factors, including VEGF, IL-8 and bFGF, are regulated in tumor cells by epidermal growth factor receptor (EGFR) signaling, which plays an important role in tumorigenesis $(25,26)$. In the EGFR family, the overexpression of ErbB-2 also leads to the increased expression of angiogenic factors, whereas treatment with anti-EGFR or anti-ErbB-2 agents produces a significant reduction in the synthesis of these proteins by cancer cells (25). In our previous study, ErbB-2 was found to be activated in HCC cell lines and tissues. We determined that the inhibition of ErbB-2 by an ErbB-2 targeting drug, trastuzumab, retarded the tumor development from HCC cells (27). Therefore, these results suggest that the identification of IL-8 and bFGF may be valuable as downstream targets of EGFR for the treatment of individual patients with HCC.

In conclusion, our findings showed that the upregulation of either IL-8 or bFGF is closely related to HCC development from liver cirrhosis. The results may be helpful in studying whether IL-8 and bFGF could be promising targets for anti-angiogenic therapies. The feasibility of utilizing protein arrays in this study suggests that arrays can be a useful tool for detecting the expression of angiogenic factors contributing to hepatocarcinogenesis and thereby identifying novel therapies for HCC.

\section{References}

1. Ferlay J, Shin HR, Bray F, Forman D, Mathers C and Parkin DM: Estimates of worldwide burden of cancer in 2008: GLOBOCAN 2008. Int J Cancer 127: 2893-2917, 2010.

2. Blum HE: Hepatocellular carcinoma: therapy and prevention. World J Gastroenterol 11: 7391-7400, 2005.

3. Portolani N, Coniglio A, Ghidoni S, et al: Early and late recurrence after liver resection for hepatocellular carcinoma: prognostic and therapeutic implications. Ann Surg 243: 229-235, 2006.

4. Poon RT and Fan ST: Resection prior to liver transplantation for hepatocellular carcinoma: a strategy of optimizing the role of resection and transplantation in cirrhotic patients with preserved liver function. Liver Transpl 10: 813-815, 2004. 
5. Grepin R and Pages G: Molecular mechanisms of resistance to tumour anti-angiogenic strategies. J Oncol 2010: 835680, 2010.

6. Sanz-Cameno P, Trapero-Marugan M, Chaparro M, Jones EA and Moreno-Otero R: Angiogenesis: from chronic liver inflammation to hepatocellular carcinoma. J Oncol 2010: 272170, 2010

7. Naumov GN, Folkman J and Straume O: Tumor dormancy due to failure of angiogenesis: role of the microenvironment. Clin Exp Metastasis 26: 51-60, 2009.

8. Zappala G, McDonald PG and Cole SW: Tumor dormancy and the neuroendocrine system: an undisclosed connection? Cancer Metastasis Rev 32: 189-200, 2013.

9. Hisai H, Kato J, Kobune M, et al: Increased expression of angiogenin in hepatocellular carcinoma in correlation with tumor vascularity. Clin Cancer Res 9: 4852-4859, 2003.

10. Pang R and Poon RT: Angiogenesis and antiangiogenic therapy in hepatocellular carcinoma. Cancer Lett 242: 151-167, 2006.

11. Bradford MM: A rapid and sensitive method for the quantitation of microgram quantities of protein utilizing the principle of protein-dye binding. Anal Biochem 72: 248-254, 1976.

12. Crowther JR: ELISA. Theory and practice. Methods Mol Biol 42: $1-218,1995$.

13. Moserle L and Casanovas O: Anti-angiogenesis and metastasis: a tumour and stromal cell alliance. J Intern Med 273: 128-137, 2013.

14. Rak J and Klement G: Impact of oncogenes and tumor suppressor genes on deregulation of hemostasis and angiogenesis in cancer. Cancer Metastasis Rev 19: 93-96, 2000.

15. Sun HC and Tang ZY: Angiogenesis in hepatocellular carcinoma: the retrospectives and perspectives. J Cancer Res Clin Oncol 130: 307-319, 2004.

16. Schwartz M, Roayaie S and Konstadoulakis M: Strategies for the management of hepatocellular carcinoma. Nat Clin Pract Onco 4: 424-432, 2007.

17. Qazi BS, Tang K and Qazi A: Recent advances in underlying pathologies provide insight into interleukin-8 expression-mediated inflammation and angiogenesis. Int J Inflam 2011: 908468, 2011.
18. Teicher BA: Antiangiogenic agents and targets: A perspective. Biochem Pharmacol 81: 6-12, 2011.

19. Hussain F, Wang J, Ahmed R, et al: The expression of IL-8 and IL-8 receptors in pancreatic adenocarcinomas and pancreatic neuroendocrine tumours. Cytokine 49: 134-140, 2010.

20. Arenberg DA, Kunkel SL, Polverini PJ, Glass M, Burdick MD and Strieter RM: Inhibition of interleukin-8 reduces tumorigenesis of human non-small cell lung cancer in SCID mice. J Clin Invest 97: 2792-2802, 1996.

21. Moore BB, Arenberg DA, Stoy K, et al: Distinct CXC chemokines mediate tumorigenicity of prostate cancer cells. Am J Pathol 154: 1503-1512, 1999.

22. Ishii Y, Sakamoto T, Ito R and Yanaga K: Anti-angiogenic therapy on hepatocellular carcinoma development and progression. J Surg Res 158: 69-76, 2010.

23. Poon RT, Ng IO, Lau C, Yu WC, Fan ST and Wong J: Correlation of serum basic fibroblast growth factor levels with clinicopathologic features and postoperative recurrence in hepatocellular carcinoma. Am J Surg 182: 298-304, 2001.

24. Yoshiji H, Kuriyama S, Yoshii J, et al: Synergistic effect of basic fibroblast growth factor and vascular endothelial growth factor in murine hepatocellular carcinoma. Hepatology 35: 834-842, 2002.

25. De Luca A, Carotenuto A, Rachiglio A, et al: The role of the EGFR signaling in tumor microenvironment. J Cell Physiol 214: 559-567, 2008.

26. Normanno N, Bianco C, De Luca A, Maiello MR and Salomon DS: Target-based agents against ErbB receptors and their ligands: a novel approach to cancer treatment. Endocr Relat Cancer 10: 1-21, 2003.

27. Liu S, Gong J, Morishita A, et al: Use of protein array technology to investigate receptor tyrosine kinases activated in hepatocellular carcinoma. Exp Ther Med 2: 399-403, 2011. 